\title{
Pre Eclampsia: Its Effect on Renal, Cardiovascular, Hepatic and Thyroid Functions a Review
}

\author{
Sabitha kandi ${ }^{1}$, B Venu Gopal ${ }^{1}$, Raj Kumar G $^{1}$, MD Rafi ${ }^{1}$, T Sudhakar ${ }^{1}$, C Ramadevi ${ }^{2}$, Mamatha ${ }^{2}$, Pragna Rao ${ }^{3}$, \\ K V Ramana, ${ }^{4, *}$ \\ ${ }^{1}$ Department of Biochemistry, Chalmeda Anandrao Institute of Medical Sciences, Karimnagar, India \\ ${ }^{2}$ Department of Obstetrics and Gynaecology, Chalmeda Anandrao Institute of Medical Sciences, Karimnagar, India \\ ${ }^{3}$ Department of Biochemistry, Kasturba Medical College, Manipal University, Manipal, India \\ ${ }^{4}$ Department of Microbiology, Prathima Institute of Medical Sciences, Karimnagar, India \\ *Corresponding author: ramana_20021@rediffmail.com
}

Received April 14, 2014; Revised December 15, 2014; Accepted December 22, 2014

\begin{abstract}
Preeclampsia is a severe disorder of pregnancy characterized by the development of hypertension and proteinuria, increased vascular resistance and endothelial dysfunction in the mother, altered placental perfusion and restricted fetal growth. There is increased release of soluble growth factor like sFlt 1 from placenta, which decreases the availability of Vascular Endothelial Growth Factor(VEGF) receptors and decreases the availability of endothelial Nitric Oxide synthase. This inturn decreases the diastolic relaxation and endothelial dysfunction, podocyte injury of glomerular cells, increased excretion of podocyte specific proteins in urine leads to proteinuria. Cardiac hypertrophy further complicates the heart function. There is a raise in liver function; soluble factor like tyrosine kinase 1 (sFlt 1) may cause a disturbance in thyroid hormone function, thus prone to thyroid problem during or after pregnancy.
\end{abstract}

Keywords: preeclampsia, proteinuria, cardiac hypertrophy, soluble factor like tyrosine kinase 1 (sFlt 1), endothelial dysfunction

Cite This Article: Sabitha kandi, B Venu Gopal, Raj Kumar G, MD Rafi, T Sudhakar, C Ramadevi, Mamatha, Pragna Rao, and K V Ramana, "Pre Eclampsia: Its Effect on Renal, Cardiovascular, Hepatic and Thyroid Functions a Review.” American Journal of Clinical Medicine Research, vol. 2, no. 6 (2014): 111-113. doi: 10.12691/ajcmr-2-6-2.

\section{Introduction}

Preeclampsia is an idiopathic multisystem disorder specific to pregnancy. It is characterized by hypertension (Blood pressure $>140 / 90 \mathrm{mmHg}$ ), Proteinuria $(>=$ $300 \mathrm{mg} / 24$ hours) and pathologic oedema during gestation $[1,2]$. The risk factors include nulliparity, multifetal gestations, obesity, diabetes mellitus, vascular and connective tissue disorders like systemic lupus erythematosus and anti-phospholipid antibodies, age $>35$ years at first pregnancy, smoking. Among the primi women the risk of African American women is twice that of Caucasian women and the risk is high in Indian and Pakistan women [3]. The Placenta is the most central organ in the pathogenesis of pre eclampsia, release of soluble factors from the ischemic placenta into maternal plasma plays a central role in endothelial dysfunction that is the most prominent feature of this disease [4]. The cause for preeclampsia is not clear but thought to be due to excess levels of soluble fms- like tyrosine kinase 1 than normal seen during pregnancy $[5,6]$. The placenta makes soluble factor like tyrosine kinase 1 (sFlt 1) in large amounts, but circulating mononuclear cells have also been shown to be an extra source of sFlt 1 in preeclampsia [7,8]. It is associated with increased maternal morbidity and mortality. The development of chronic hypertension, cardiovascular and renal complications may be seen in later part of life in preeclamptic individuals [9]. The vasospasm and endothelial injury in multiple organs are the results of pre eclampsia. These soluble factors sFlt 1 decreases or inhibits the binding of Vascular Endothelial Growth Factor(VEGF) to its receptors which in turn reduces the phosphorylation of endothelial nitricoxide synthase (eNOS) leading to endothelial dysfunction [10]. The placental secretions are in excess containing factors like tyrosine kinase 1 which may contribute to the endothelial dysfunction, hypertension, proteinuria [11]. During implantation, insufficient placental circulation leads to Pregnancy Induced Hypertension (PIH). This poor circulation may produce proinflammatory molecules which injures, mothers endothelial cells results in increased vascular resistance. A functional imbalance in Thrombaxane A2 (TXA2) and prostacyclin play a role in development of preeclampsia. TXA2 acts via Neurogenin - B, downregulates VEGF - mediated signaling [12].

In late pregnancy (age $>35$ ) the placenta release increased sFlt -1 , decreases VEGF mediated signaling. Decreased VEGF activity impairs diastolic relaxation [13). Detecting proteinuria is an integral part of the management of hypertensive pregnant women and is associated with higher rate of maternal and foetal complications among hypertensive pregnancies [14]. 


\section{Renal Changes due to Preeclampsia}

The Vascular endothelial growth factor (VEGF) plays a critical role in maintenance of normal glomerular endothelial integrity. VEGF actions even though maintained by protein tyrosine kinase activity, the increased sFlt 1 inturn decreases the availability of receptors for VEGF. Thus, causes endothelial dysfunction [15]. The proteinuria seen in women with preeclampsia is due to podocyte injury, and reduced specific podocyte protein expression. The urinary excretion of podocyte specific proteions (podocalyxin, nphrin \& Big - h3) was significantly elevated in preeclampsia [16].

The reduced renal perfusion and glomerular filtration is secondary to renal dysfunction. Soluble endoglin (sEng) is another antiangiogenic factor isolated from from placenta and blood of women with preeclampsia, it inhibits binding of transforming growth factor (TGF) $\beta$ to its receptors and cause down regulation of nitric oxide synthase [17]. The combination of raised sFlt 1 and sEng impairs nitric oxide generation and activate endothelin signaling pathway [18]. It may be due to damage caused by endothelin, a potent endogenous vasoconstrictor peptide produced by endothelial cells [19].

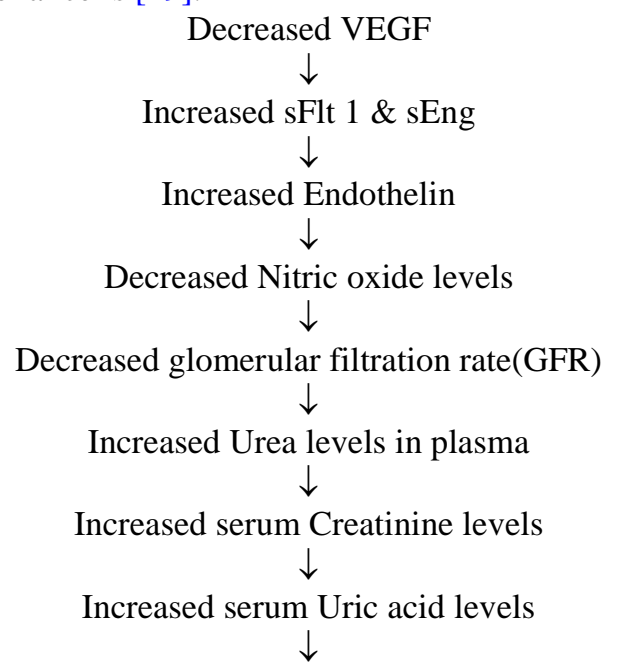

Due to decreased clearance of these substances [20,21,22,23]

Thus, hyperuricemia of pregnancy is of particular interest to obstetrician because of its association with preeclampsia [24].

\section{Cardiovascular Changes due to Preclampsia}

The cardiovascular system undergoes a host of changes with the development of preeclampsia. The diastolic dysfunction was observed more frequently in preeclampsia than in normal pregnancy. The diastolic dysfunction may lead to ischemic / hypertensive cardiac diseases and is of prognostic value in predicting long term cardiovascular morbidity [25]. Left ventricular hypertrophy is commonly seen in pregnancy due to volume overload which is reversible after pregnancy. The hemodynamic changes of preeclampsia make these cardiac hypertrophy $(\mathrm{CH})$ irreversible. Cardiac hypertrophy is a complex process that involve change in the composition of extracellular matrix such as fibrosis, changes in contractility and fetal gene expression, as well as altered angiogenesis [26].
Micro RNAs are non - coding RNAs that regulate gene expression by blocking transcription or by repressing translation of their target genes $[27,28]$. Micro RNA plays a key role in the development of hypertrophy. miR -1 \& $\mathrm{miR}-133$ that are related to regulate growth genes are responsible for cardiac hypertrophy [29,30].

The sex hormones especially estrogens has a role in cardiac hypertrophy of preeclampsia [31] as estrogen levels are raised in late pregnancy. The Ubiquitin Protease (UP) enzymes are involved in degradation of misfolded protein. In $\mathrm{CH}$ there is increased denovo synthesis of proteins, there may be misfolding of protein or abnormal protein synthesis. Hence UP activity is enhanced to check for any misfold or abnormal protein synthesis. In preeclampsia also there is enhanced UP activity due to pressure overload [32].

\section{Hepatic Changes due to Preclampsia}

In pre eclampsia there are mild disturbances in liver function and histology. However the hepatic complications are minor. Liver dysfunction is characterized by development of pain in right upper quadrant or epigastric pain [33]. The Alkaline phosphatase enzyme (ALP) levels are raised during the third trimester of pregnancy, these increased ALP has no physiological function and is metabolically inert [34]. In pre-eclamptic pregnancy the ALP levels were significantly raised than that in non pregnant controls [35]. The enzyme Gamma-Glutamyl Transpeptidase (GGT) levels are also raised in all hypertensive pregnancy [36].

\section{The Thyroid Hormone Changes due to Pre Eclampsia}

The cause of preeclampsia known from experimental studies both from humans and animals suggests, excess release of anti-angiogenic factors such as soluble fms like tyrosine kinase1 (sFlt -1 / s VEGFR1) from placenta to maternal blood. The levels of these sFlt -1 increases during last two months of normal pregnancy. There levels are very much increased in women with preeclampsia. In normal pregnancy there is increased Thyroid stimulating hormone (TSH) due to raised human chorionic gonadotropin (hCG) concentration [37]. But in pre eclampsia the raised TSH is not due to change in hCG but the raised TSH is directly related to the circulating levels of sFlt -1 concentrations. This suggests that the effect of pre eclampsia on thyroid function is mediated by sFlt -1 [38]. These sFlt -1 acts by inhibiting Vascular Endothelial Growth Factor (VEGF) and placental growth factor signaling $[39,40]$. The renal podocytes require constitutive expression of VEGF for health and function. The reduced level of VEGF makes the endothelium of glomerular capillaries to be fenestrated [41] and may lead to proteinuria of preeclampsia. Thyroid capillaries also have a fenestrated epithelium [42], which in turn increases the secretion of TSH concentrations. Thus, high levels of exposure to sFlt -1 as in preeclampsia may be associated with increased risk for reduced thyroid function during and after pregnancy. 


\section{Conclusion}

Thus, preeclampsia has to be carefully monitored as it may contribute to complications both to mother and fetus. Genetic causes, hypothyroid conditions, liver and kidney function, cardiovascular functions are to be regularly checked not only during pregnancy but also after delivery to reduce the resultant morbidity and mortality.

\section{References}

[1] Wagner L.K. Diagnosis and Management of pre eclampsia, American Family Physicians; 2004: 70(12): 2318-2324.

[2] Prakash J, Pandey L.K, Singh A.K and Kar B. Hypertension in pregnancy, Hospital based study, JAPI; 2006:54:273-278.

[3] A.K. Rao, K. Daniels, Y. Y. El - Sayed, M. K. Moshesh, and A. B. Caughey, " Perinatal outcomes among Asian American and Pacific Islander women”. American Journal of Obstetrics and Gynecology, vol. 195, no. 3, pp. 834-838, 2006.

[4] Elosha Eiland, Chike Nzerue, and Marquetta Faulkner, Preeclampsia 2012, Journal of Pregnancy, vol.2012.

[5] Maynard Se, Min JY, Merchan J, Lim KH, Li J, Mondal S et. Al. Excess placental soluble fms - like tyrosine kinase $1(\mathrm{sFlt}-1)$ may contribute to endothelial dysfunction, hypertension and proteinuria in pre eclampsia. J Clin Invest; 2003;111:649-58.

[6] Levine Rj, Maynard SE, Qian C, Lim KH, England LJ, Yu KF et.al., Circulating angiogenic factors and the risk of pre eclampsia. N Engl J Med 2004; 350:672-83.

[7] D. Hui, N. Okun, K. Murphy, J. Kingdom, E. Uleryk, and P.S. Shah, "Combinations of maternal serum markers to predict preeclampsia, small for gestational age, and stillbirth: a systematic review”., Journal of Obstettrics and Gynaecology Cananda, vol.34, no.2, pp. 142-153, 2012.

[8] S. Venkatesha, M. Toporsian, C. Lam et al., "Soluble endoglin contributes to the pathogenesis of preeclampsia", Nature Medicine, vol.12, no.6, pp. 642-649, 2006.

[9] Sibai B.M and Gonzalez R.A. Severe pre eclampsia - eclampsia in young primigravid women : Subsequent pregnancy outcome and remote prognosis, Am Journal Obstet Gynaecol, 1986: 155: 10111016.

[10] D. S. Gelinas, P. N. Bernatchez, S. Rollin, N. G. Bazan, and M. G. Sirois, "Immediate and delayed VEGF - mediated NO synthesis in endothelial cells: role of P13K, PKC and PLC pathways”, British Journal of pharmacology, vol. 137. No. 7, pp. 1021-1030, 2002.

[11] Robert L. Barbieri, John T. Repke. Medical disorders during pregnancy: Harrison's principles of internal medicine: chapter 6: volume I: Mc Graw Hill Publishing division; 2005: 32.

[12] Furuya M, Ishida J, Aoki I, Fukamizu A. Pathophysiology of placentation abnormalities in pregnancy - induced hypertension. Vasc Health Risk Manag. 2008; 4: 101-1313.

[13] Patten IS, Rana S, Shahul S, Rowe GC, Jang et.al.,. Cardiac angiogenic imbalance leads to peripartum cardiomyopathy. Nature. 2012; 485: 333-338.

[14] A. Wright, British Journal of Obstetrics and Gynecology 1987; 94: 408-412.

[15] I. E. Stillman and S. A Karumanchi, "The glomerular injury of preeclampsia”, Journal of the American Society of Nephrology, vol.18, no. 8, pp. 2281-2284, 2007.

[16] Y Wang, S.Zhao, S.Loyd and L. J. Groome, "Increased urinary excretion of nephrin, podocalyxin, and big - h3 in women with preeclampsia”, American Journal of physiology, vol.30, no.9, pp. F1084 - F1089, 2012.

[17] Q. Chen, L. Chen, B, Liu et al. "The role of autocrine TGF $\beta 1$ in endothelial cell activation induced by phagocytosis of necrotic trophoblasts; a possible role in the pathogenesis of pre eclampsia”, Journal of pathology, vol.221, no.1,pp.87-95, 2010.

[18] V.C. Sandrim, A. C. T. Palei, I. F. Metzger, V. A. Gomes, R. C. Cavalli, and J. E. Tanus - Santos, "Nitric oxide formation is inversely related to serum levels of antiangiogenic factors soluble fms - like tyrosine kinase 1 and soluble endogline in preeclampsia”, Hypertension, vol.52, no. 2, pp. 402-407, 2008.

[19] Slowinsk i, T. Neumayer, H.H: stolze, T, Grossing G; Halle, H and Houcher,B 2002. Endothelin system in normal and hypertensive pregnancy, clinical science.103(48); 446s-449.

[20] Hassan TJ, Jaerey SN, J Pak Med Assoc. 1991; 41(8): 183-5.

[21] Williams Obstetrics $21^{\text {st }}$ edition.

[22] Barbara A. Clark, MD, Lisa Halvorson, MD. Am J Obstet Gynecol : 1992; 166: 962-968.

[23] Lionel J.schewitz. The medical clinics of north America.

[24] British journal of obstetrics and gynaecology, 1997; 84: 13-21.

[25] Melchiorre K, Sutherland GR, Baltabaeva A, Liberati M, Thilaganathan B. 'Maternal cardiac dysfunction and remodeling in women with preeclampsia at term'. Hypertension. 2011 Jan; 57(1) 85-93.

[26] Mann DL, Bristow MR. mechanisms and models in heart failure: the biomechanical model and beyond. Circulation. 2005; 111: 2837-849.[pub med].

[27] Bartel DP, Chen CZ. Micromanagers of gene expression: the potentially widespread influence of metazoan mocroRNAs. Nat Rev Genet. 2004; 5: 396-400. [pub med].

[28] Bartel DP, Micro RNAs: target recognition and regulatory functions. Cell. 2009; 136: 215=233. [PMC free article] [pub med]

[29] Sayed D, Hong C, Chen IY, Lypowy J, Abdellatif M. MicroRNAs play an essential role in the development of cardiac hypertrophy. Circ res. 2007; 100: 416-424. [pub med].

[30] Care A, Catalucci D, Felicetti F, Bonci D, Addario A, et al., Micro RNA - 133 controls cardiac hypertrophy. Nat Med 2007; 13: 613-618. [pub med].

[31] Gardner JD, Murray DB, Voloshenyuk TG, Brower GL, Bradley JM et al.,. Estrogen attenuates chronic volume induced structural and functional remodeling in male rat hearts. Am J Physiol Heart Circ Physiol. 2010;298: H497 -H504. [PMC free article] [pub med].

[32] Otsuka K, Terasaki F, shimomura H, Tsukada B, Horii T, Isomura $\mathrm{T}$ et al.,. Enhanced expression of the ubiquitin - proteasome system in the myocardium from patients with dilated cardiomyopathy referred for left ventriculoplasty: an immunohistochemical study with special reference to oxidative stress. Heart vessels. 2010; 25: 474-484. [pub med]

[33] Burrow and Duffy : Medical complications during pregnancy.

[34] The new England journal of medicine jan27, 1972.

[35] Journal of Obstetrics and Gynecology ; 1984; 91: 857-862.

[36] Churchill D1, Kilby MD, Bignell A, Whittle MJ, Beevers DG Gamma-glutamyl transferase activity in gestational hypertension. Br J Obstet Gynaecol. 1994 Mar; 101(3): 251-3.

[37] 37. Larijani B, Marsoosi V, Aghakhanl S, Moradi A, Hashmipour S. Thyroid hormone alteration in pre-eclamptic women. Gynecol Endocrinol 2004; 18: 97-100.

[38] 38. Richard J Levine, lars J Vatten, Gary L Horowitz,Cong Qian, Pal R Romundstad et al., Pre eclampsia, soluble fms - like tyrosine kinase 1 , and the risk of reduced thyroid function: nested case control and population based study. BMJ 2009; 339: b4336.

[39] Eremina V, Jefferson JA, Kowalewska J, Hochster H, Haas M, et al., VEGF inhibition and renal thrombotic micro angiopathy. $\mathrm{N}$ Engl J Med 2008; 358: 1129-36.

[40] Kamba T, Mc Donald DM. Mechanism of adverse effects of anti VEGF therapy for cancer 2007; 96: 1788-95.

[41] Risau W. Development and differentiation of endothelium. Kidney Int 1998; 67: s3-6.

[42] Kamba T, Tam BY, Hashizume H, Haskell A, Sennino B et al., VEGF dependent plasticity of fenestrated capillaries in the normal adult microvasculature. Am J Physiol Heart irc Physiol 206; 290: H560-76. 